\title{
Closed-form response of a linear fractional visco-elastic oscillator under arbitrary stationary input
}

\author{
V. Denoëla,
}

${ }^{a}$ Structural Engineering Division, Faculty of Applied Sciences, University of Liège, Liège, Belgium

\begin{abstract}
This paper studies the structural response of a single degree-of- freedom structure including a fractional derivative constitutive term. Unlike usual existing models for this kind of structure, the excitation is not necessarily a Markovian process but it is slowly varying in time, so that a timescale separation is used. Following the general formulation of the Multiple Timescale Spectral Analysis [1], the solution is developed as a sum of background and resonant components. Because of the specific shape of the frequency response function of a system equipped with a fractional viscoelastic device, the background component is not simply obtained as the variance of the loading divided by the stiffness of the system. On the contrary the resonant component is expressed as a simple extension of the existing formulation for a viscous system, at least at leading order. As a validation case, the proposed solution is shown to recover similar results (in the white noise excitation case) as former studies based on a stochastic averaging approach [2,3,4]. A better accuracy is however obtained in case of very small fractional exponent. Another example related to the buffeting analysis of a linear fractional viscoelastic system demonstrates the accuracy of the proposed formulation for colored excitation.
\end{abstract}

Keywords: Caputo fractional derivative, Riemann-Liouville fractional derivative, perturbation analysis, stochastic averaging, background component, resonant component

\section{Fractional Calculus in Mechanics}

Fractional calculus has attracted considerable attention over the last decades in the mechanical and structural engineering community. In some early works [5] dealing with the modeling of visco-elastic 1 damping, a fractional derivative Maxwell model was used. An experimental demonstration [6] has confirmed the appropriateness of this model and further trigger scientific curiosity about fractional calculus in mechanics. This model is based on the Riemann-Liouville definition of the fractional derivative operator

$$
\mathcal{D}^{\alpha} y(t)=\frac{1}{\Gamma(1-\alpha)} \int_{0}^{t} \frac{\dot{y}(\tilde{t})}{(t-\tilde{t})^{\alpha}} d \tilde{t}
$$

where $\alpha \in[0,1]$ is the fractional exponent. Some other experimental investigations and identification techniques have shown that this model is able to accurately capture the dynamics of visco-elastic systems $[7,8,9]$. Also the linearity of this operator (the fractional derivative of a linear combination is the linear combination of the fractional derivatives) makes it rather attractive to combine viscoelastic devices with others existing features of linear stochastic dynamics

Email address: v.denoel@ulg.ac.be (V. Denoël)
$[10,11,2,12,13]$. The nonstationary solution of linear systems might be expressed in closed form [14], which constitutes another reason to use this type of model. As soon as nonlinear stochastic dynamical systems are considered, the exact solution is usually not obtained in closed form, even for wide-band (usually white noise) excitation. Approximations similar to or derived from the stochastic linearization and stochastic averaging methods [15, 16, 17, 18, 19], or those based on a Fokker-Planck equation of the process envelope [20] appear to be the most classical ways to deal with such problems. Narrow band excitations and complex dynamical interactions can be simplified with similar multiple scales approaches [21]. Other more realistic types of loadings [22] or even earthquake loadings of linear and nonlinear systems equipped with viscoelastic devices have also been considered in $[23,24]$.

Beside these approximations of the exact solution of the problem, other numerical techniques have been proposed to deal with the structural analysis of systems with fractional derivatives. In particular, Monte Carlo simulation methods are consistently used to validate approximations. Also there exist ad hoc simulation methods [14] which are computationally efficient, and methods based on Wiener path integral approaches $[25,26,27,28]$. These techniques, together with exact assembling procedures of structural analysis [29], or finite element approaches [30, 4, 12], 

viscoelastic devices.

This review of the literature reveals two trends. On 82 one side, there are simple dynamical systems with 8 linear (or linearized) behaviour and white noise ex- 84 citation, which possess closed form solutions. On 85 the other side, there are numerical techniques to deal 86 with more realistic loadings (sometimes nonstation- ${ }_{87}$ ary), more realistic structures and/or slight nonlin- 88 earities. The missing gap in-between is related to the $\mathbf{8 9}$ understanding (via simple analytical solutions) of the 90 behavior of complex structures subjected to more re- 91 alistic loadings. As a first step towards this goal, 92 we consider the stochastic analysis of a linear system ${ }_{93}$ subjected to a low frequency loading specified by its 94 arbitrary power spectral density. More precisely, we consider the oscillator governed by

$$
x^{\prime \prime}(\tau)+2 \xi \mathcal{D}^{\alpha} x(\tau)+x(\tau)=u(\tau)
$$

where $\xi$ is the dimensionless fractional coefficient and 99 $u(\tau)$ is a stationary random process with an arbitrary 100 power spectral density $S_{u}(\Omega)$ with the restriction ${ }^{101}$ that its characteristic timescale is slow compared to ${ }^{102}$ the dimensionless natural frequency of the oscillator ${ }^{103}$ (which is equal to unity). This problem is a dimen- ${ }^{104}$ sionless version of a classical singe degree-of-freedom ${ }^{105}$ system with given mass, stiffness and damping, see 106 [31] for the details of the scaling. This equation is for 107 instance encountered in the analysis of wind-excited 108 structures with visco-elastic devices, where the ob- 109 jective is to determine the statistics (the variance, ${ }^{110}$ mainly) of the structural response. The fact that the ${ }^{111}$ exogenous loading $u(\tau)$ could in principle be a non ${ }^{112}$ Markovian process is a specific contribution of this ${ }^{113}$ study.

This problem could be studied by means of the ${ }_{115}$ usual time domain multiple scales approach, or the ${ }_{116}$ stochastic averaging approach, but would require the ${ }_{117}$ consideration of three interacting timescales as well as ${ }_{118}$ fractional models - similar to those used to simulate ${ }_{119}$ realizations of wind fields [32],- - for the augmented ${ }_{120}$ state coloring the excitation. This seems possible to ${ }_{121}$ solve the problem in this way, although no track ev- ${ }_{122}$ idences of this type of problem has been found in ${ }_{123}$ the literature. Instead, we take advantage of the lin- ${ }_{124}$ earity of the problem to derive a simplified solution ${ }_{125}$ in a frequency domain. It is based on the Multiple ${ }_{126}$ Timescale Spectral Analysis [1] which seeks the same ${ }_{127}$ objectives as the stochastic averaging, with slightly ${ }_{128}$ more versatility as shown next.

\section{The Multiple Timescale Spectral Analysis ${ }^{131}$}

In 1961, Davenport proposed a method to com- ${ }_{133}$ pute the response of structures to the buffeting ac- ${ }^{134}$ tion of wind [33], which is known today as the 135 Background/Resonant decomposition [34]. In this 136 method, the total variance of the response, which corresponds to the area under the power spectral density of the response a sketched in Figure 3, is approached by the sum of two terms. The first one, the background component, corresponds to the quasi-static response of the structure and therefore depends on the stiffness of the system. The second one, the resonant component, corresponds to the resonance of the structure and is also governed by the damping in the system. It is possible to prove that this decomposition is valid in the necessary and sufficient condition that the two timescales associated with the problem of buffeting, i.e. the slow action of wind and the fast dynamics of the structure, are well separated. With the usual rules of perturbation theories, this translates into one order of magnitude at least [35].

In fact, it turns out that the decomposition proposed by Davenport in 1961 is just a particular case of the Multiple Timescales Spectral Analysis, which has been recently formalized by the author [1]. Under this general terminology, one can find an ad hoc version of the computation of integrals with small parameters, as typically depicted in Pertubation Methods textbooks, e.g. [35]. Indeed, in many applications of stochastic dynamics, the integrals to be computed (in the frequency domain) feature several small parameters. In particular, as soon as the ratio of two of the timescales of a problem is a small number, the problem is said to feature well separated timescales. Although this ratio is the most important small number, there are several additional small numbers in engineering applications: small damping, small nonlinearity, small stochasticity (see for instance [36] where four small numbers are identified and their smallness is exploited to construct very accurate approximate solutions of the problem).

The Multiple Timescale Spectral Analysis (MTSA) is a method to develop simple analytical solutions, or at least to reveal the influence of the different problem parameters to the various contributions to the stochastic response of a system. As stated by its name, the MTSA requires the existence of various well separated timescales in the problem. We notice that this does not really limit the possible field of application and corresponds anyways to usual assumptions formulated in deterministic and stochastic averaging [37]. The MTSA consists in recognizing the existence of these different timescales and consider the problem with all these timescales, successively, and provide local approximations in the frequency domain for the statistical moments associated with all of them. The method is rather general and is not limited to two timescales, nor to the estimation of the variance of the response. It has already been applied to the estimation of covariances of modal responses in case of multi-degree of freedom structures [38] (although this was before the general method has been fully formalized). In this context, the MTSA provides 


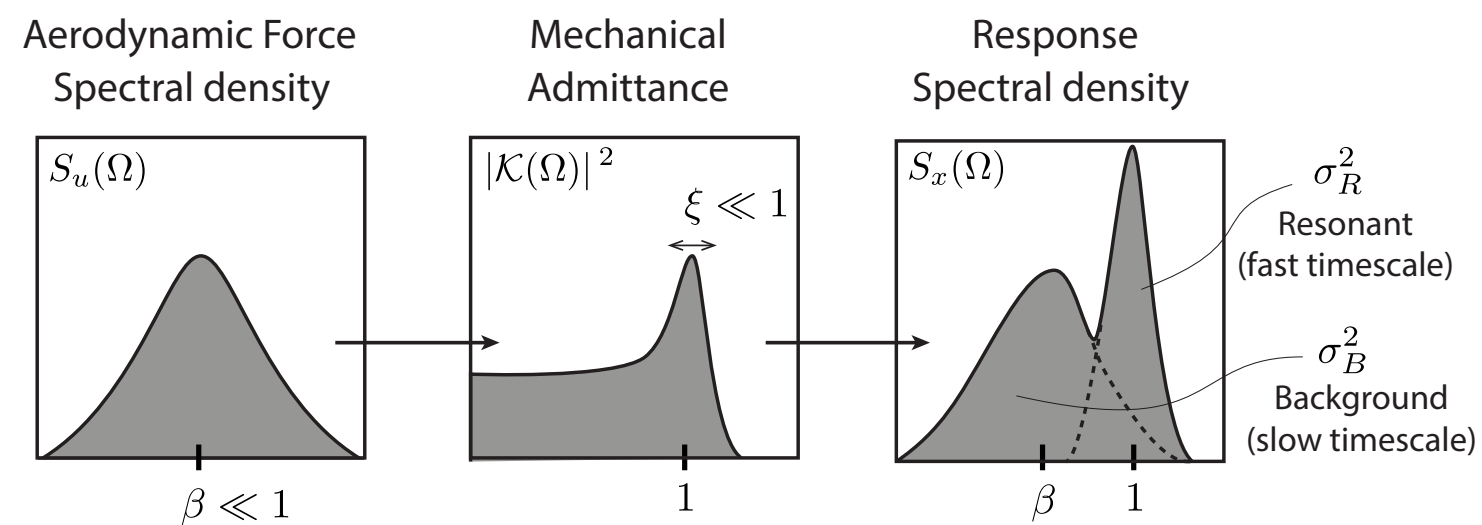

Figure 1: Sketch illustrating the decomposition of the response to buffeting into a slow background component and a fast resonant component [33].

\section{Solution of The Problem}

In this paper, the Multiple Timescales Spectral Analysis is applied to the considered problem, which demonstrates the ability of the method to deal with visco-elastic dampers, but also to provide an engineering insight into the different components of the structural response of a stochastically excited oscillator in the presence of dissipative devices modeled with fractional derivatives. To do so, let us first con- 188 sider the Fourier transform of (2)

$$
\left(1+\left.2 \xi \mathcal{C} \Omega\right|^{\alpha}-\Omega^{2}-\left.2 \xi i \mathcal{S} \Omega\right|^{\alpha}\right) X(\Omega)=U(\Omega)
$$

where $\mathcal{C}=\cos \frac{\alpha \pi}{2}$ and $\mathcal{S}=\sin \frac{\alpha \pi}{2}$, so that the power spectral density of the response is given by

$$
\begin{aligned}
S_{x}(\Omega) & =\frac{S_{u}(\Omega)}{\left(1+2 \xi \mathcal{C}|\Omega|^{\alpha}-\Omega^{2}\right)^{2}+\left(2 \xi \mathcal{S}|\Omega|^{\alpha}\right)^{2}} \\
& :=K(\Omega) S_{u}(\Omega) .
\end{aligned}
$$

This equation defines the kernel $K(\Omega)$. The only assumption required for the method to be applicable is that the central frequency $\beta$ in the loading $S_{u}(\Omega)$ be much smaller that unity, $\beta \ll 1$ i.e. that the stochastic loading is slower that the dynamics of the system. This kernel is illustrated in Figure 2 for several values of $\alpha$. It has some peculiarities: (i) the resonance peak located near $\Omega= \pm 1$ in the viscous case $(\alpha=1)$ regularly moves to higher frequencies as $\alpha \rightarrow 0$, i.e. as the fractional derivative term tends to correspond to a stiffness term. In the limiting case $\alpha=0$, the fractional derivative corresponds to a usual stiffness term and the peak is located at abscissa $\Omega_{p}=\sqrt{1+2 \xi} \simeq 1+\xi$; (ii) the frequency response function passes trough a common crossing point, at abscissa $\Omega=1$, no matter the fractional exponent $\alpha$; (iii) the intercept is $K(0)=1$ provided $\alpha \neq 0$. As $\alpha \rightarrow 0$, a short boundary layer, whose extent is of order $\alpha$, develops in the neighborhood of the origin and creates the transition from the upper bound $K(0)=1$ to the lower bound $K(\Omega) \simeq \frac{1}{(1+2 \xi)^{2}}$. For $\alpha \rightarrow 0$, the size of this transition zone tends to zero; for $\alpha=0$, there is no transition anymore and $K(0)=\frac{1}{(1+2 \xi)^{2}}$.

As a result of the fractional powers of $\Omega$ appearing in $K(\Omega)$, the response of the system at second order, its variance, defined as

$$
\sigma_{x}^{2}=\int_{-\infty}^{+\infty} S_{x}(\Omega) d \Omega,
$$

is unfortunately not available in a simple closed form, even for simple forms of $S_{u}(\Omega)$. This is all the more valid for complex expressions of $S_{u}(\Omega)$ corresponding non Markovian processes. 


\section{$K(\Omega)$}

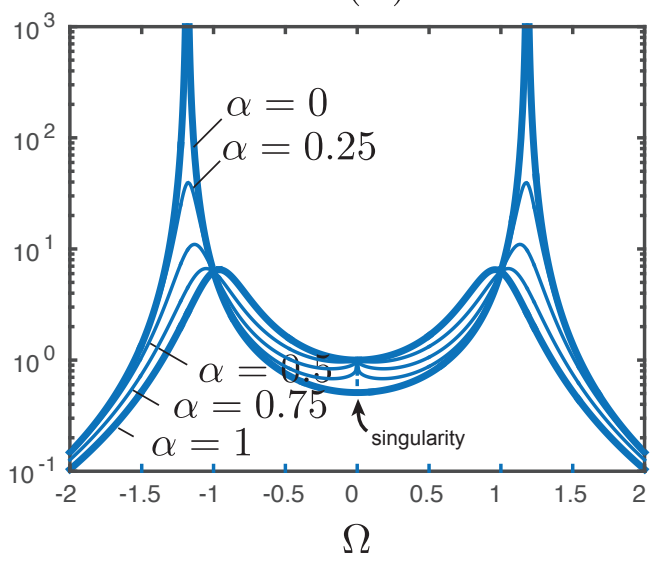

Figure 2: Representation of the kernel $K(\Omega)$ for various values of the fractional exponent $\alpha$. Other parameters: $\xi=0.2$.

Figure 3 shows some examples of the power spectral density of the structural response given by (4). This function features two distinct types of peaks: one in the low-frequency range around $\Omega \simeq 0$ and over a domain whose extent is of order $\beta$ (the background component) and the other in the order-one frequency range (the resonant component). They are well distinct because $\beta \ll 1$.

Application of the Multiple Timescale Spectral Analysis consists in successively focusing on the different contributions to the response. In this application, there are only 2 components.

First, the background component is evaluated by rescaling the frequency axis $\Omega$ with the stretched coordinate $\zeta$ defined as $\Omega=\beta \zeta$. With this scaling, the ${ }^{215}$ background component develops over a domain of or- ${ }^{210}$ der 1 and, considering the separation of timescales $\beta \ll 1$, the kernel $K[\Omega(\zeta)]$ can be approximated by

$$
\hat{K}[\Omega(\zeta)]=\frac{1}{1+4 \xi \mathcal{C} \beta^{\alpha}|\zeta|^{\alpha}+4 \xi^{2} \beta^{2 \alpha}|\zeta|^{2 \alpha}} .
$$

This is the frequency response function of a lowpass fractional filter [43]. The expression $S_{u}(\Omega) \hat{K}(\Omega)$ is therefore a local approximation of $S_{x}(\Omega)$ in the neighborhood of the origin, for $\Omega \sim \beta \ll 1$. This approximation is represented by dotted lines in Figure 3, for $\alpha=0.25$ and $\xi=0.2$. Using this approximation, the background component of the response is expressed as

$$
\begin{aligned}
\sigma_{x, b}^{2} & =\int_{-\infty}^{+\infty} S_{u}(\Omega) \hat{K}(\Omega) d \Omega \\
& =\int_{-\infty}^{+\infty} \frac{S_{u}(\Omega)}{1+4 \xi \mathcal{C}|\Omega|^{\alpha}+4 \xi^{2}|\Omega|^{2 \alpha}} d \Omega
\end{aligned}
$$

which is the lowpass fractional filtered energy in the loading. For $\alpha \simeq 1$ and $\xi \ll 1$, the frequency response ${ }_{223}$ function of this filter tends to unity and
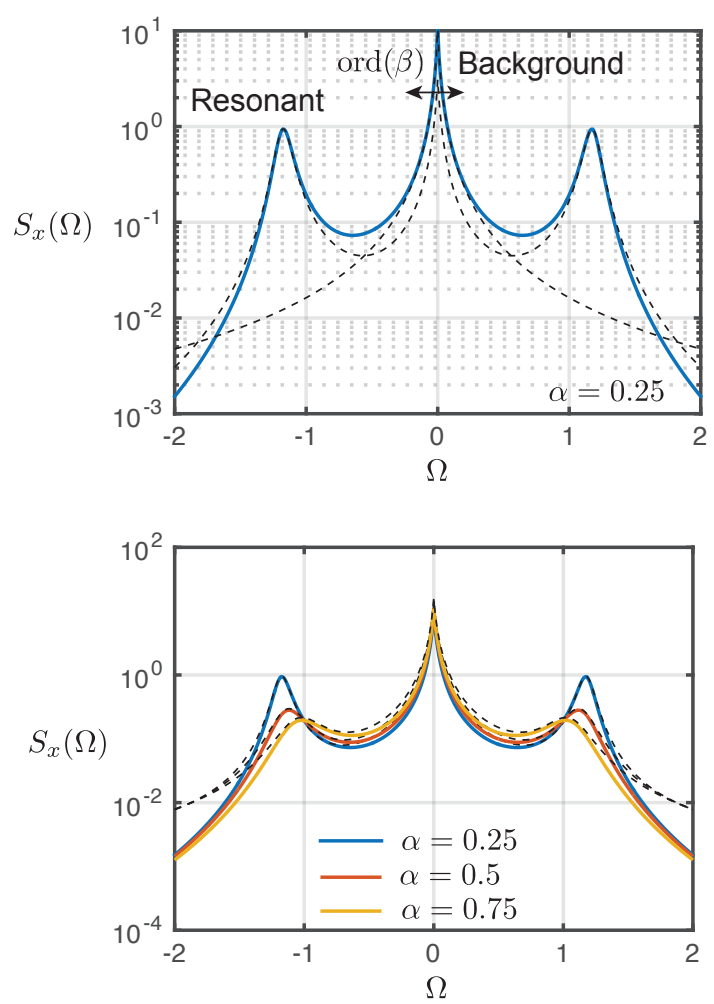

Figure 3: Examples of the power spectral density of the structural response for various values of the fractional exponent $\alpha$. Other parameters: $\xi=0.2, \beta=0.05$.

$$
\lim _{\alpha \rightarrow 1, \xi \rightarrow 0} \sigma_{x, b}^{2}=1
$$

which is the well-known result from linear stochastic dynamics.

Second, the resonant component needs to be developed. To do so a remainder is constructed by subtracting this first approximation $\hat{K}(\Omega)$ from the original function to integrate, that is

$$
\begin{aligned}
r_{1} & =\int_{-\infty}^{+\infty} S_{x}(\Omega)-S_{u}(\Omega) \hat{K}(\Omega) d \Omega \\
& =\int_{-\infty}^{+\infty} S_{u}(\Omega)(K(\Omega)-\hat{K}(\Omega)) d \Omega .
\end{aligned}
$$

The function to be integrated features two symmetrical peaks which will equally contribute the resonant part of the response. So we only only focus on the positive peak, then multiply by two. It is possible to prove [31] that the peaks (in absolute value) are located close to abscissa

$$
\Omega_{p}=1+\mathcal{C} \xi-\left[\alpha+\left(\frac{1}{2}-\alpha\right) \mathcal{C}^{2}\right] \xi^{2}+\mathcal{O}\left(\xi^{2}\right)
$$

The position of the peak is a perturbation of 1 (the dimensionless natural frequency) and 
To summarize, the background/resonant decomposition of the variance of a linear oscillator with fractional derivatives is given by

$$
\begin{aligned}
& \sigma_{x}^{2}=\sigma_{x, b}^{2}+\sigma_{x, r}^{2}= \\
& \quad \int_{-\infty}^{+\infty} \frac{S_{u}(\Omega)}{1+4 \xi \mathcal{C}|\Omega|^{\alpha}+4 \xi^{2}|\Omega|^{2 \alpha}} d \Omega+\frac{\pi S_{u}(1+\mathcal{C} \xi)}{2 \mathcal{S} \xi \sqrt{1+2 \xi \mathcal{C}}} .
\end{aligned}
$$

This expression regularly extends the well-known background resonant decomposition in case of integer derivative $(\alpha=1, \mathcal{C}=0, \mathcal{S}=1)$, which is

$$
\lim _{\alpha \rightarrow 1} \sigma_{x}^{2}=1+\frac{\pi S_{u}(1)}{2 \xi} .
$$

It also shows that, at leading order, the response of a fractionally damped oscillator depends on the power spectral density of the loading, computed for a unique value of the frequency: $\Omega=1+\mathcal{C} \xi$. This is the only way the response depends on the power spectral density of the loading. With this approximation, we show that the Markovianity of the input of this system is secondary; in other words, the proposed solution is valid no matter the shape of the power spectral density (rational fractions of $\Omega$ or not).
2 263 264 264 266 267 267 268 269 270

\section{Validation, illustrations and discussion}

In order to validate the proposed solution, we determine the response of the oscillator subjected to a loading specified by

$$
S_{u}(\Omega)=\frac{0.546}{\beta\left(1+1.64 \frac{|\Omega|}{\beta}\right)^{5 / 3}}
$$

with $\beta \ll 1$ is a small dimensionless characteristic frequency. In wind engineering applications, it is related to the slow turbulence, compared to the natural frequency of the structure. The accuracy of the proposed formulation is assessed by comparison with a reference result obtained by accurate numerical integration of the exact power spectral density of the response. Integration is performed with the adaptive algorithm proposed in Wolfram Mathematica [44], with default integration parameters of Version 11.0.1.0.

Figures 4 and 5 show the variance of the response obtained with the proposed formulation (MTSA) and by numerical integration of the exact analytical formulation (Exact). The variance is represented as a function of $\alpha$ for given values of $\xi$, and as a function of $\xi$ for given values of $\alpha$. It is also given for two values of $\beta$, namely $\beta=0.01$ and $\beta=0.1$. These two small numbers correspond to typical values of this parameter in buffeting applications.

In all cases, the proposed formulation (MTSA) provides very accurate results, when compared to the reference solution (Exact). The smaller $\beta$, the more accurate. This is consistent with the methodology to develop the approximate solution and with the inherent assumptions in the Multiple Timescale Spectral Analysis. The same observation also holds for $\xi$ which also needs to be a relatively small number. In fact, this relative smallness can be discussed with this example. Indeed, the comparison shows that the proposed method is very accurate for values of $\xi$ which are as large as 1.

In both figures, the background component $\sigma_{x, b}^{2}$ is shown with dashed lines. Where the total variance is similar to background component $\sigma_{x, b}^{2}$, the resonant counterpart to the response is negligible and the response is actually quasi-static. This happens for large values of the damping (see Figure 4) or, for given $\xi$, 

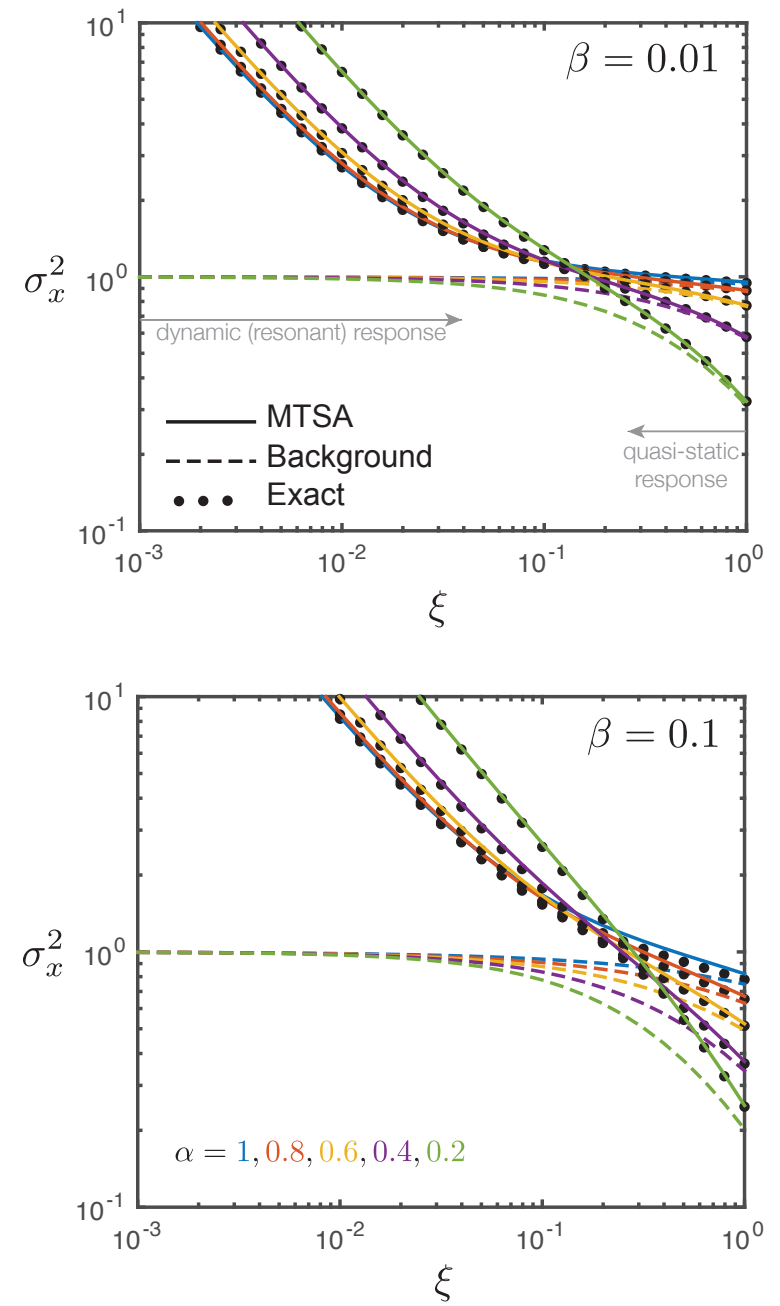

Figure 4: Variances of the response of the system subjected to the buffeting type excitation, for $\beta=0.01$ (top) and $\beta=0.1$ (bottom). Represented as a function of the fractional coefficient $\xi$. and for various values of the fractional exponent $\alpha$ (Please see online version for colors)
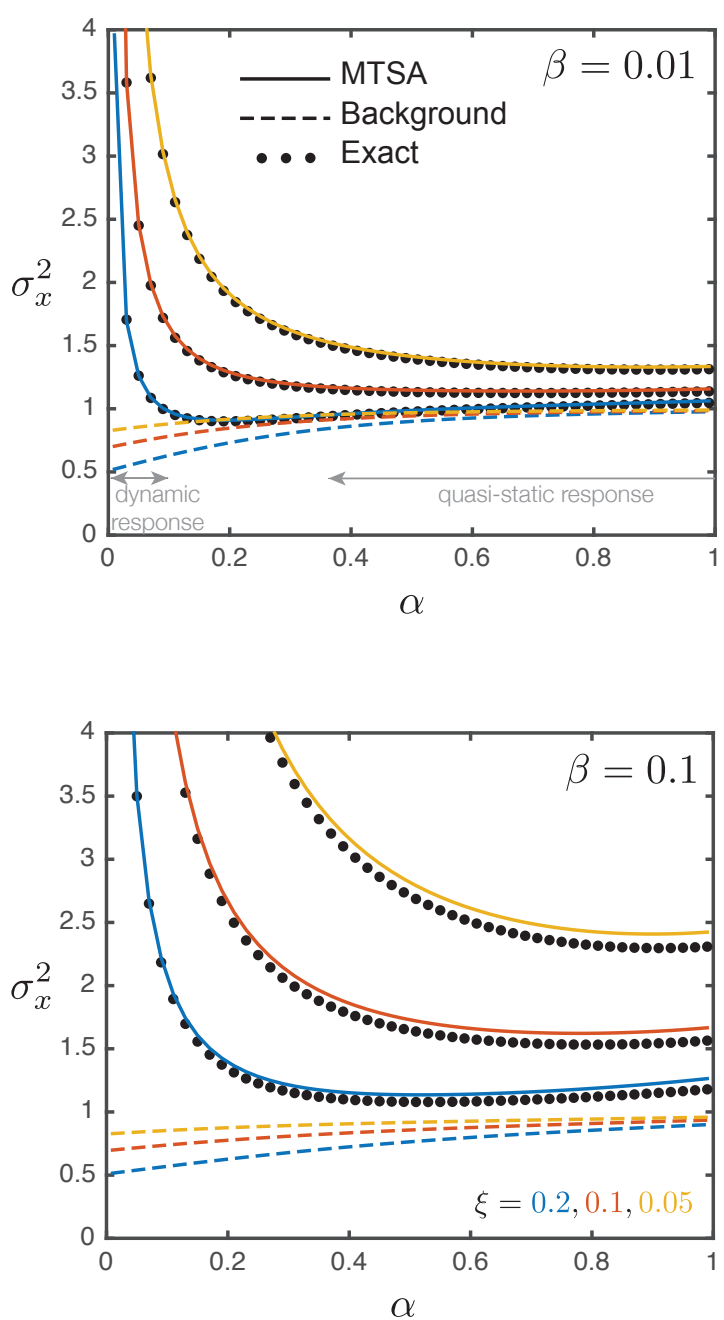

Figure 5: Variances of the response of the system subjected to the buffeting type excitation, for $\beta=0.01$ (top) and $\beta=0.1$ (bottom). Represented as a function of the fractional exponent $\alpha$ and for various values of the fractional coefficient $\xi$. (Please see online version for colors) 
for large values of $\alpha$, i.e. as the behaviour of the $\mathbf{3 5 2}$ fractional damper tends to viscosity (see Figure 5). ${ }^{353}$

\section{Conclusions}

In this paper, we have applied the Multiple ${ }^{358}$ Timescale Spectral Analysis to the structural anal- 360 ysis of a linear system equipped with a viscoelastic ${ }^{\mathbf{3 6 1}}$ device. The proposed formulation extends the well ${ }_{363}^{362}$ known background/resonant decomposition which is ${ }_{364}$ usually applied in the field of wind engineering. It $\mathbf{3 6 5}$ shows that

- the background component can be determined ${ }^{368}$ without any prior knowledge of the dynamics of ${ }^{369}$ the system; it consists in a fractional filtered ver- 371 sion of the input;

- the power spectral density of the loading just ${ }^{374}$ enters in the solution of this problem through the ${ }_{376}^{375}$ value of the power spectral density at a frequency 377 equal to $\Omega=1+\cos \frac{\alpha \pi}{2} \xi$;

- the resonant component of the response $\sigma_{x, r}^{2}$ is ${ }^{380}$ proportional to the power spectral density; $\quad{ }_{382}^{\mathbf{3 8 1}}$

- the resonant component of the response $\sigma_{x, r}^{2}$ does ${ }_{384}^{383}$ not scale with the inverse of the damping ratio ${ }^{385}$ anymore (as what would be obtained in the in- ${ }^{386}$ teger derivative case). It rather scales with the ${ }^{387}$ inverse of $\xi \sqrt{1+2 \xi \mathcal{C}}$. In the limit case $\alpha \rightarrow 1_{389}{ }^{388}$ (viscous damping), $\mathcal{C} \rightarrow 0$, the usual scaling is $\mathbf{3 9 0}$ recovered

- the resonant component scales with $\mathcal{S}^{-1}={ }^{393}$ $\csc \frac{\alpha \pi}{2}$, which tends to infinity as $\alpha \rightarrow 0$. This ${ }_{395}^{394}$ results from the fact that there is no damping 396 anymore in this limit case, and the dynamic re- ${ }^{397}$ sponse is unbounded.

These preliminary results are very promising. Future ${ }_{401}^{400}$ works should combine the developments summarized 402 in this paper with other contexts of application of the ${ }^{403}$ Multiple Timescale Spectral Analysis and consider in ${ }_{404}^{404}$ this way slightly nonlinear systems (with the help of ${ }_{406}^{405}$ a Volterra model) or to multiple degree-of-freedom 407 structures equipped with fractional derivative dissi- ${ }^{408}$ pative devices.

\section{References}

[1] Vincent Denoël. Multiple timescale spectral analysis. ${ }_{\mathbf{4 1 5}}^{\mathbf{4 1 4}}$ Probabilistic Engineering Mechanics, 39(0):69-86, jan 416 2015.

[2] P. D. Spanos and B. A. Zeldin. Random vibration of ${ }_{\mathbf{4 1 8}}$ systems with frequency-dependent parameters or frac- $4 \mathbf{4 1 9}$ tional derivatives. Journal of Engineering Mechanics, 420 123(3):290-292, 1997.

[3] A. Palmeri, F. Ricciardelli, G. Muscolino, and A. De Luca. Random vibration of systems with viscoelastic 423 memory. Journal of Engineering Mechanics, 130(9):1052- 424 1061, 2004.
[4] Mario Di Paola, Antonina Pirrotta, Francesco Paolo Pinnola, and Salvatore Di Lorenzo. Stochastic response of fractionally damped beams. Probabilistic Engineering Mechanics, 35:37-43, 2014.

[5] R L Bagley and P J Torvik. Fractional calculus in the transient analysis of viscoelastically damped structures. AIAA Journal, 23(6):918-925, jun 1985.

[6] Nicos Makris and M. C. Constantinou. Fractionalderivative maxwell model for viscous dampers. Journal of Structural Engineering, 117(9):2708-2724, 1991.

[7] Wei Sun, Zhuo Wang, Xianfei Yan, and Mingwei Zhu. Inverse identification of the frequency-dependent mechanical parameters of viscoelastic materials based on the measured frfs. Mechanical Systems and Signal Processing, 98:816 - 833, 2018.

[8] Roman Lewandowski and Bartosz Chorazyczewski. Identification of parameters of the fractional maxwell model. 102018.

[9] Mario Di Paola, A Pirrotta, and A Valenza. Visco-elastic behavior through fractional calculus: an easier method for best fitting experimental results. Mechanics of Materials, 43(12):799-806, 2011.

[10] O.P. Agrawal. Stochastic analysis of dynamic systems containing fractional derivatives. Journal of Sound and Vibration, 247(5):927 - 938, 2001.

[11] Mircea Grigoriu. Linear systems with fractional brownian motion and gaussian noise. Probabilistic Engineering Mechanics, 22(3):276 - 284, 2007.

[12] Mikael Enelund and B Lennart Josefson. Time-Domain Finite Element Analysis of Viscoelastic Structures with Fractional Derivatives Constitutive Relations. AIAA Journal, 35(10):1630-1637, oct 1997.

[13] Sami I Muslih and Dumitru Baleanu. Hamiltonian formulation of systems with linear velocities within riemannliouville fractional derivatives. Journal of Mathematical Analysis and Applications, 304(2):599-606, 2005.

[14] Mario Di Paola, Giuseppe Failla, and Antonina Pirrotta. Stationary and non-stationary stochastic response of linear fractional viscoelastic systems. Probabilistic Engineering Mechanics, 28:85 - 90, 2012.

[15] Lincong Chen, Weihua Wang, Zhongshen Li, and Weiqiu Zhu. Stationary response of duffing oscillator with hardening stiffness and fractional derivative. International Journal of Non-Linear Mechanics, 48:44 - 50, 2013.

[16] Z. L. Huang, X. L. Jin, C. W. Lim, and Y. Wang. Statistical analysis for stochastic systems including fractional derivatives. Nonlinear Dynamics, 59(1):339-349, Jan 2010.

[17] Yongge Yang, Wei $\mathrm{Xu}$, Xudong Gu, and Yahui Sun. Stochastic response of a class of self-excited systems with caputo-type fractional derivative driven by gaussian white noise. Chaos, Solitons and Fractals, 77:190 - 204, 2015.

[18] Yongge Yang, Wei Xu, Wantao Jia, and Qun Han. Stationary response of nonlinear system with caputo-type fractional derivative damping under gaussian white noise excitation. Nonlinear Dynamics, 79(1):139-146, Jan 2015.

[19] Giuseppe Failla and Antonina Pirrotta. On the stochastic response of a fractionally-damped duffing oscillator. Communications in Nonlinear Science and Numerical Simulation, 17(12):5131-5142, 2012.

[20] Jun-Feng A Zhao. Stochastic response of dynamical systems with fractional derivative term under wide-band excitation. Mathematical Problems in Engineering, page 9, 2016.

[21] Yong Xu, Yongge Li, and Di Liu. Response of Fractional Oscillators With Viscoelastic Term Under Random Excitation. Journal of Computational and Nonlinear Dynamics, 9(3):31015-31018, feb 2014.

[22] L.C. Chen, Q.Q. Zhuang, and W.Q. Zhu. Response of sdof nonlinear oscillators with lightly fractional derivative damping under real noise excitations. The European Physical Journal Special Topics, 193(1):81-92, Mar 2011. 
23] Ye Kun, Li Li, and Tang Jiaxiang. Stochastic seismic 498 response of structures with added viscoelastic dampers 499 modeled by fractional derivative. Earthquake Engineering $\mathbf{5 0 0}$ and Engineering Vibration, 2(1):133-139, Jun 2003.

4] Pol D. Spanos and Georgios I. Evangelatos. Response of a 502 non-linear system with restoring forces governed by frac- 503 tional derivatives: Time domain simulation and statisti- 504 cal linearization solution. Soil Dynamics and Earthquake 505 Engineering, 30(9):811 - 821, 2010.

[25] Guy Jumarie. Path integral for the probability of the 507 trajectories generated by fractional dynamics subject $\mathbf{5 0 8}$ to gaussian white noise. Applied Mathematics Letters, 509 20(8):846 - 852, 2007.

26] Ioannis A Kougioumtzoglou, Alberto Di Matteo, Pol D 511 Spanos, Antonina Pirrotta, and Mario Di Paola. An Ef- 512 ficient Wiener Path Integral Technique Formulation for $\mathbf{5 1 3}$ Stochastic Response Determination of Nonlinear MDOF Systems. Journal of Applied Mechanics, 82(10):101005101007, jul 2015.

[27] A. Lotfi and S.A. Yousefi. A numerical technique for solv- ${ }^{514}$ ing a class of fractional variational problems. Journal of Computational and Applied Mathematics, 237(1):633 - 643, 2013.

[28] Alberto Di Matteo, Ioannis A. Kougioumtzoglou, Antonina Pirrotta, Pol D. Spanos, and Mario Di Paola. Stochastic response determination of nonlinear oscillators with fractional derivatives elements via the wiener path integral. Probabilistic Engineering Mechanics, 38:127 - 135, 2014.

[29] Giuseppe Failla. Stationary response of beams and frames with fractional dampers through exact frequency response functions. Journal of Engineering Mechanics, 143(5):D4016004, 2017.

[30] Gioacchino Alotta, Giuseppe Failla, and Massimiliano Zingales. Finite-element formulation of a nonlocal hereditary fractional-order timoshenko beam. Journal of Engineering Mechanics, 143(5):D4015001, 2017.

[31] Vincent Denoël. Multiple timescale spectral analysis of a linear fractional viscoelastic system under colored excitation. Probabilistic Engineering Mechanics, 53:66-74, 2018.

[32] Giulio Cottone and Mario Di Paola. Fractional spectral moments for digital simulation of multivariate wind velocity fields. Journal of Wind Engineering and Industrial Aerodynamics, 99(6):741 - 747, 2011. The Eleventh Italian National Conference on Wind Engineering, INVENTO-2010, Spoleto, Italy, June 30th - July 3rd 2010.

[33] A G Davenport. The application of statistical concepts to the wind loading of structures. Proceedings of the Institute of Civil Engineers, 19:449-472, 1961.

[34] EN. EN 1991 Eurocode 1: Actions on structures - Part 14: General actions - Wind actions, Brussels, 2005. CEN.

[35] E J Hinch. Perturbation Methods, volume 1. Cambridge University Press, Cambridge, 1991.

[36] Vincent Denoël and Luigi Carassale. Response of an oscillator to a random quadratic velocity-feedback loading. Journal of Wind Engineering and Industrial Aerodynamics, 147:330 - 344, 2015.

[37] John Brian Roberts and Pol D Spanos. Random vibration and statistical linearization. Courier Corporation, 2003.

[38] Vincent Denoël. Estimation of modal correlation coefficients from background and resonant responses. Structural Engineering and Mechanics: an International Journal, 32(6):725-740, 2009.

[39] Edward L Wilson, Armen Der Kiureghian, and EP Bayo. A replacement for the srss method in seismic analysis. Earthquake Engineering $\&$ Structural Dynamics, 9(2):187-192, 1981.

[40] Vincent Denoël. On the background and biresonant components of the random response of single degree-offreedom systems under non-Gaussian random loading. Engineering Structures, 33(8):2271-2283, 2011.

[41] Thomas Canor, Luca Caracoglia, and Vincent Denoël.
Perturbation methods in evolutionary spectral analysis for linear dynamics and equivalent statistical linearization. Probabilistic Engineering Mechanics, 46:1 - 17, 2016.

[42] Vincent Denoël. Extension of davenport's background/resonant decomposition for the estimation of higher response moments. In Proceedings of the Sixth European-African Conference on Wind Engineering, page 4, 2013.

[43] V. Valimaki and T. I. Laakso. Principles of fractional delay filters. In 2000 IEEE International Conference on Acoustics, Speech, and Signal Processing. Proceedings (Cat. No.00CH37100), volume 6, pages 3870-3873 vol.6, 2000.

[44] Wolfram Research, Inc. Mathematica, Version 11.0. Champaign, IL, 2016. 